SUBJECT AREAS: TUMOUR SUPPRESSORS CELL BIOLOGY

Received

3 June 2014

Accepted

31 July 2014

Published

18 August 2014

Correspondence and requests for materials should be addressed to N.T. (ntsuchiy@ncc. go.jp)

\title{
NEK9-dependent proliferation of cancer cells lacking functional p53
}

\author{
Daisuke Kurioka ${ }^{1,8}$, Fumitaka Takeshita ${ }^{2}$, Koji Tsuta ${ }^{5}$, Hiromi Sakamoto ${ }^{3}$, Shun-ichi Watanabe ${ }^{6}$, \\ Kenii Matsumoto ${ }^{7}$, Masatoshi Watanabe ${ }^{8}$, Hitoshi Nakagama ${ }^{4}$, Takahiro Ochiya ${ }^{2}$, Jun Yokota ${ }^{1,9}$, \\ Takashi Kohno' \& Naoto Tsuchiya'
}

\begin{abstract}
'Division of Genome Biology, National Cancer Center Research Institute, ${ }^{2}$ Division of Molecular and Cellular Medicine, National Cancer Center Research Institute, ${ }^{3}$ Division of Genetics, National Cancer Center Research Institute, ${ }^{4}$ Division of Cancer Development System, National Cancer Center Research Institute, ${ }^{5}$ Division of Pathology and Clinical Laboratories, National Cancer Center Hospital, 5-1-1, Tsukiji, Chuo-ku Tokyo 104-0045, 'D Division of Thoracic Surgery, National Cancer Center Hospital, 5-1-1 Tsukiji, Chuo-ku Tokyo 104-0045, ${ }^{2}$ Division of Allergy \& Immunology, National Research Institute for Child Health and Development, 2-10-1 Okura Setagaya-ku Tokyo, 157-8535, ${ }^{8}$ Laboratory for Medical Engineering, Division of Materials and Chemical Engineering, Graduate School of Engineering, Yokohama National University, 79-1 Tokiwadai, Hodogaya-ku Yokohama 240-8501, Japan, ${ }^{9}$ Institute of Predictive and Personalized Medicine of Cancer (IMPPC), Barcelona, Spain.
\end{abstract}

Dysfunction of the p53 network is a major cause of cancer development, and selective elimination of p53-inactivated cancer cells therefore represents an ideal therapeutic strategy. In this study, we performed a microRNA target screen that identified NEK9 (NIMA-related kinase 9) as a crucial regulator of cell-cycle progression in p53-inactivated cancer cells. NEK9 depletion selectively inhibited proliferation in p53-deficient cancer cells both in vitro and in vivo. The resultant cell-cycle arrest occurred predominantly in G1 phase, and exhibited senescence-like features. Furthermore, NEK9 repression affected expression of a broad range of genes encoding cell-cycle regulators and factors involved in mRNA processing, suggesting a novel role for NEK9 in p53-deficient cells. Lung adenocarcinoma patients with positive staining for NEK9 and mutant p53 proteins exhibited significantly poorer prognoses, suggesting that expression of both proteins promotes tumor growth. Our findings demonstrate that a novel NEK9 network regulates the growth of cancer cells lacking functional p53.

\footnotetext{
(1)
} he p53 tumor-suppressor pathway is the most important cellular network involved in preventing transformation of normal cells following exposure to various oncogenic insults ${ }^{1}$. In response to oncogenic stress, p53 activation leads to cell-cycle arrest, allowing for repair of damage and survival, or apoptosis, allowing for elimination of damaged cells, through stimulus-dependent transactivation of its target genes ${ }^{2}$. Cancer genome sequencing studies have shown that TP53 is one of the genes most frequently mutated in human cancers ${ }^{3,4}$. Thus, dysfunction of the p53 signaling pathway(s) is a major cause of tumor onset and/or progression in almost all human cancers ${ }^{5}$. Furthermore, the molecular network(s) specifically activated in p53-deficient contexts may promote proliferation of cancer cells. Several lines of evidence strongly suggest that TP53 mutations contribute to maintenance of the malignant gain-of-function phenotypes of cancer cells, including cell-cycle progression and activation of cell migration, as well as loss of wild-type tumor-suppressor functions ${ }^{6}$. In light of this novel concept, mutant p53 is an attractive target for therapeutics directed against a wide range of cancers.

MicroRNAs (miRNAs) are small non-coding RNAs that regulate gene expression at the post-transcriptional level ${ }^{7}$. Dysfunction of miRNAs is deeply involved in cancer development ${ }^{8}$. Many miRNAs are oncogenic and/or tumor-suppressive factors ${ }^{9}$; in particular, several miRNAs serve as intrinsic mediators that coordinate the p53 tumor-suppressor network in response to oncogenic stresses ${ }^{10}$. Each miRNA represses expression of a distinct set of target genes, determined in part by cellular characteristics influenced by p53 mutation status; consequently, these miRNAs induce different phenotypes in different cancer cells ${ }^{11-14}$. Thus, by exploiting their ability to affect the regulation of specific oncogenic or tumor-suppressive networks, individual miRNAs can be used as screening tools to identify therapeutic molecular targets in cancer cells.

Previously, we showed that $m i R-22$, which acts on the 53 network, induces cell death in cancer cells with wildtype p53. On the other hand, it induces cell-cycle arrest in p53-mutant cancer cells that express CDK6, CDK3, SIRT1, and HDAC4, which are critical factors involved in cell-cycle progression and potential targets of $m i R-22^{15}$. This finding led us to hypothesize that $m i R-22$ has a unique set of target genes that determine the fate of cancer 

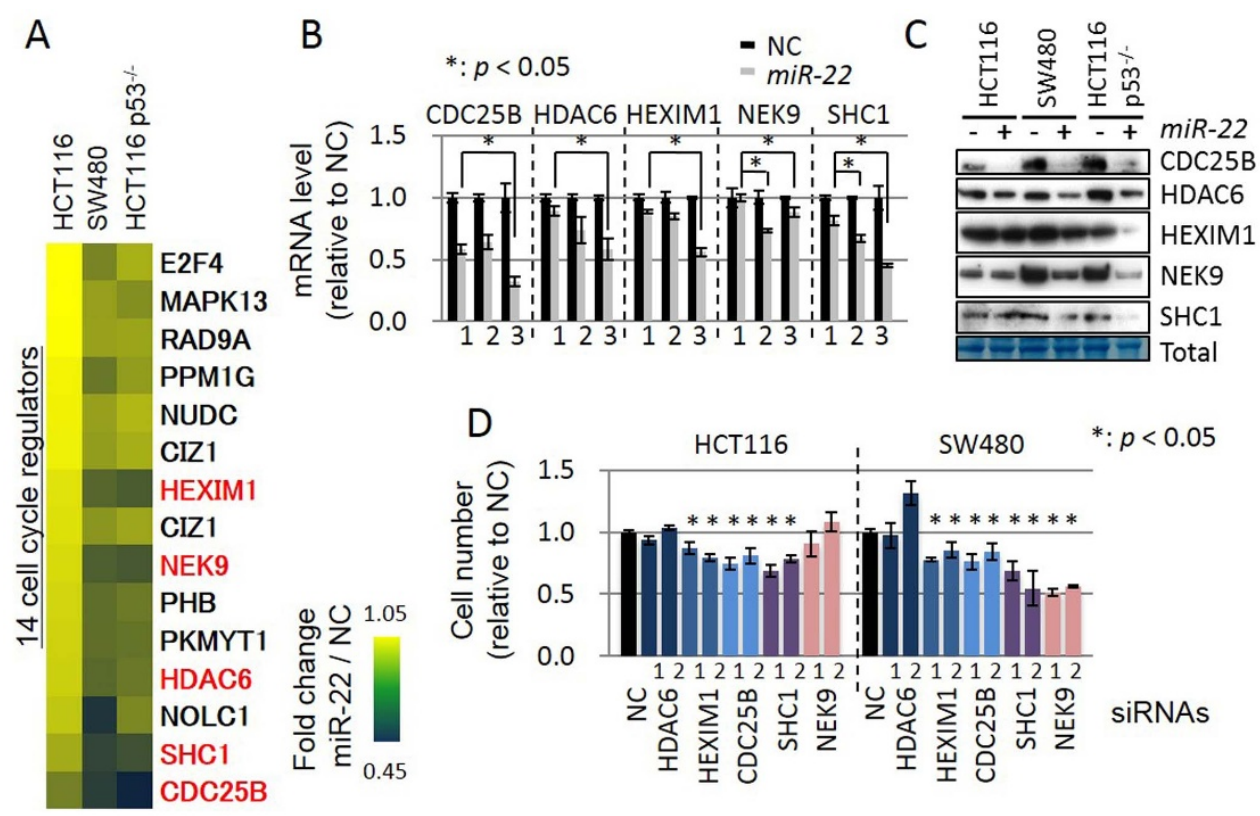

Figure 1 Identification of NEK9 by miRNA target screen. (A) Fold changes in expression ( $m i R-22 / \mathrm{NC}$ ) of 14 cell-cycle regulators, shown as a heat map. Red letters indicate potential $m i R-22$ target genes identified by in silico analysis. (B) Relative expression levels of five mRNAs identified by this screen were quantitated using TaqMan qRT-PCR assays. GAPDH was used as an internal standard. (Lane 1: HCT116; Lane 2: SW480; Lane 3: HCT116 p53 ${ }^{--}$).

(C) Protein levels of candidate genes in the presence of $m i R-22$ were determined by immunoblot analysis. A $70 \mathrm{kDa}$ protein that stained with amido black was used as a loading control. The original full scan images are available in Supplementary Figure 7. (D) Cell proliferation assay. Cells were counted $72 \mathrm{~h}$ after transfection with specified siRNAs, indicated below the graph. Two siRNAs, designated 1 and 2, were used to knock down each gene. Error bars indicate standard deviation $(\mathrm{SD}, n=3)$.

cells according to their p53 mutation status. In this study, using $m i R$ 22 as a screening tool, we tried to identify factors that play important roles in proliferation and/or cell-cycle progression of p53-mutant cancer cells. The screen identified NIMA-related kinase 9 (NEK9) as such a factor. Both in vitro and in vivo, NEK9 depletion inhibited cell growth only in p53-mutant cancer cells, and expression of the NEK9 open reading frame (ORF) in NEK9-knockdown (KD) cells restored cell proliferation. Furthermore, NEK9 KD in cancer cells induced senescence-like changes ascribed to prolonged G1 arrest or slowdown of cell-cycle progression. In addition, human lung adenocarcinoma patients with expression of both NEK9 and mutant p53 proteins exhibited significantly poorer prognoses, suggesting that NEK9 expression confers growth advantages on p53 mutant tumors. Taken together, our findings strongly suggest that the NEK9 network, which is selectively activated in p53-deficient contexts, is a crucial cellular pathway responsible for the growth capacity of cancer cells lacking functional p53.

\section{Results}

Identification of NEK9 by miRNA target screen. To identify targets selectively repressed in p53 mutant (MUT) and knockout (KO) cells, we compared changes in mRNA levels resulting from miR-22 expression between cancer cell lines with p53 KO, MUT, or wildtype (WT). As depicted in Supplementary Figure 1, we first chose the genes down-regulated in both 53 MUT and KO cells by $m i R-22$, and compared their expression levels in p53 WT cells, and then selected genes exhibiting a differential response to $m i R-22$. Furthermore, we narrowed-down the candidate genes classified as encoding cell-cycle regulators in the Gene Ontology database. The resultant 14 genes were down-regulated by $m i R-22$ expression to greater extents in p53 MUT and KO cell lines than in WT cells (Fig. 1A, Supplementary Table 1). Finally, we searched for $m i R-22$ targets among these 14 genes by in silico analysis using miRWalk database (http://www. umm.uni-heidelberg.de/apps/zmf/mirwalk/), and found that five of these genes are direct targets of $m i R-22$ (red genes in Fig. 1A).
Quantitative RT-PCR analysis confirmed that these five genes were significantly down-regulated by $m i R-22$ in both $\mathrm{p} 53 \mathrm{KO}$ and MUT cells, relative to WT cells (Fig. 1B). MiR-22 expression significantly decreased the level of NEK9 and SHC1 proteins in both p53 $\mathrm{KO}$ and MUT cells, but did not affect the levels in WT cells (Fig. 1C). Furthermore, siRNA-mediated knockdown (KD) of the five predicted $m i R-22$ targets revealed that only NEK9 depletion significantly suppressed proliferation in MUT cells; however, NEK9 KD did not affect proliferation in WT cells (Fig. 1D). Using a reportergene assay, we verified that NEK9 mRNA is a direct target of $m i R-22$ (Supplementary Figure 2). Based on these results, we concluded that NEK9 is an $m i R-22$ target gene whose repression specifically suppresses growth of p53-mutant cancer cells.

NEK9 expression is required for proliferation of p53-inactivated cancer cells. To confirm that NEK9 depletion specifically suppresses growth of p53-mutant cells, we knocked down this protein in a set of cancer cell lines with different p53 mutation status (Fig. 2A, Supplementary Figure 3A). NEK9 KD significantly suppressed the growth of three cancer cell lines with p53 mutations, but did not affect the growth of three cancer cell lines with WT p53. In four subsequent experiments, we verified that the effect of NEK9 KD depended on p53 status. First, we stably knocked down p53 in HCT116 cells, which normally express WT p53, via expression of a specific shRNA. NEK9 KD significantly decreased growth in p53 KD cells, but not in cells expressing a control shRNA (Fig. 2B, Supplementary Figure 3B). Second, we established H1299 cell lines (a p53-null lung cancer cell line) that exogenously expressed either WT p53 or one of four p53 mutants (Supplementary Figure 3C). Three of the mutants, R175H, R273H, and R249S, are frequently observed in a variety of human cancers (IARC p53 database, http://p53.iarc.fr/); the fourth, R280K, is less common. In H1299 cells expressing mutant p53, but not in those expressing WT p53, siRNA-mediated NEK9 KD significantly suppressed growth (Fig. 2C). NEK9 KD also suppressed colony formation (Fig. 2D) 


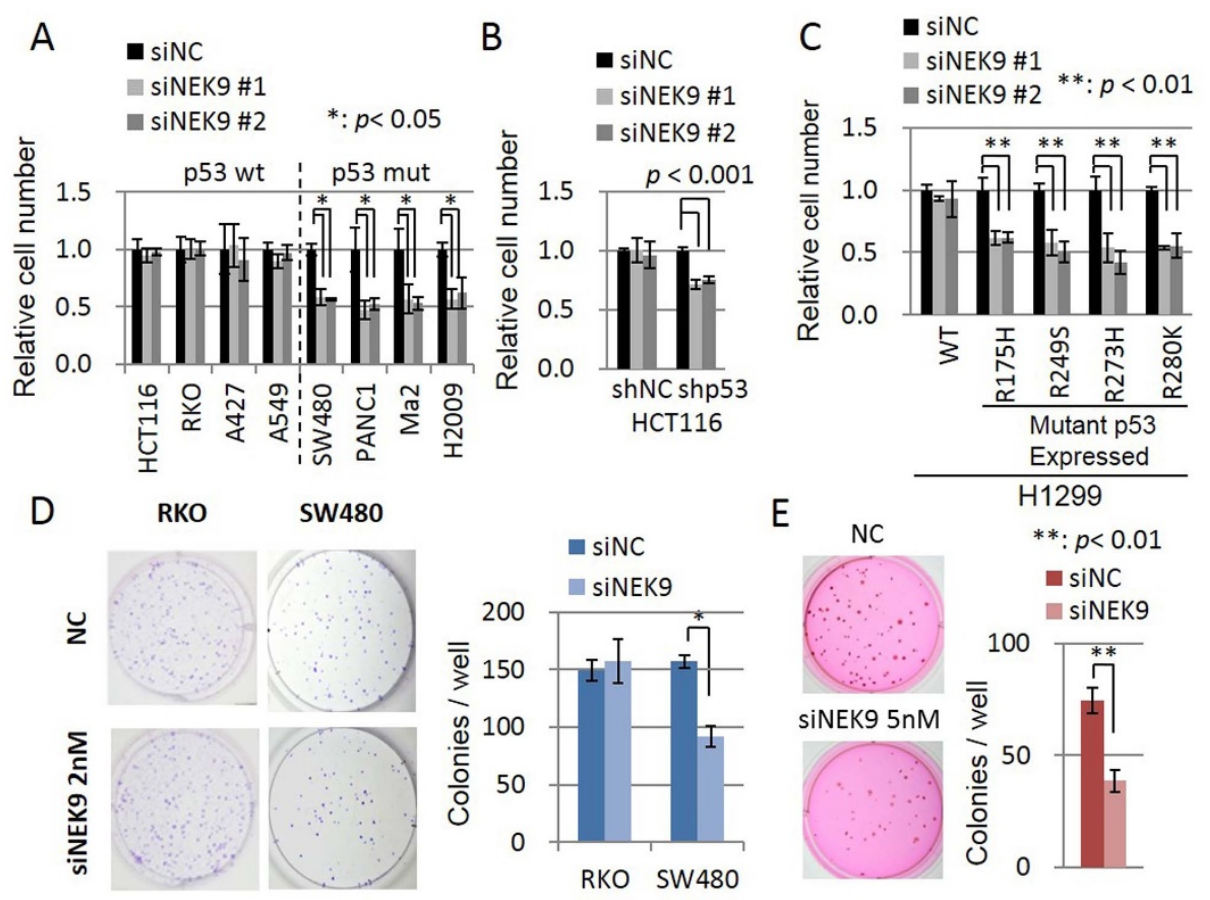

$\mathrm{F}$

G
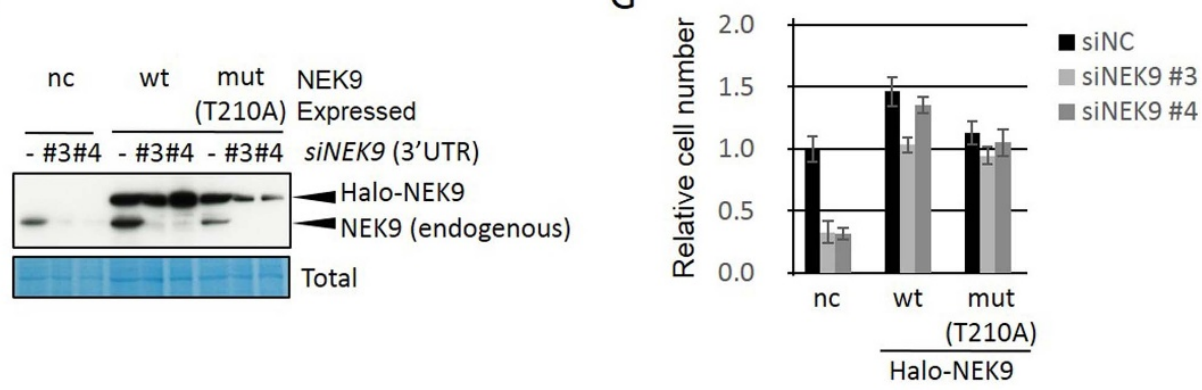

Figure $2 \mid$ Depletion of NEK9 inhibits proliferation of p53-inactivated cancer cells. (A) Cells were counted 3 days (or 4 days, for Ma2 and H2009 cells) after transfection with either 2 nM NEK9 siRNA (\#1 or \#2) or non-targeting control ( siNC). Error bars indicate SD ( $n=3$ ). (B) HCT116 cells in which p53 was stably knocked down were transfected with $5 \mathrm{nM}$ siRNAs, and the cell number was determined. (C) H1299 transfectants expressing WT p53 or one of four p53 mutants were depleted of NEK9, and then subjected to proliferation assays. Error bars indicate SD $(n=3)$. (D) Colony-formation assay on culture dishes was conducted using RKO (p53 WT) and SW480 (p53 MUT) cells, transfected with 2 nM NEK9 siRNAs or NC siRNA, and then allowed to grow for 9 days. Representative images of the colonies, stained with crystal violet, are shown on the left; colony counts are shown in the graph on the right. Three independent experiments yielded similar results; error bars indicate $\mathrm{SD}(n=3)$. (E) Images of colonies of NEK9 KD cancer cells are shown at left, and the graph indicates the number of colonies. Error bars indicate SD $(n=3)$. (F) Expression of NEK9 protein after knockdown of endogenous NEK9. SW480 cells were co-transfected with NEK9 siRNAs (\#3 and \#4) targeting the 3' UTR region of its mRNA and a siRNA-resistant NEK9 ORF expression vector. KD or expression of Halo-tagged NEK9 was determined by IB. WT and MUT (T210A) indicate wild-type and T210 mutant (kinase-inactivated) of NEK9, respectively. The original full scan images are available in Supplementary Figure 7. (G) Cell proliferation assay for NEK9 ORF-expressing cells. Cell counts are expressed relative to the count in the negative control (i.e., co-transfected with NC siRNA and vector control), which was normalized to 1 .

and anchorage-independent growth of MUT cells (Fig. 2E). Third, we asked whether NEK9 protein expression would restore cell proliferation in p53-mutant cells. Endogenous NEK9 was knocked down by siRNA targeting the 3'UTR of NEK9 mRNA, followed by expression of a cDNA encoding an siRNA-resistant NEK9 ORF (Fig. 2F), and then the cells were counted. As shown in Fig. 2G, NEK9 re-expression restored cell growth after KD of endogenous NEK9. Expression of an amino-acid-substituted form of NEK9, T210A, in which an autophosphorylation site required for kinase activity of NEK9 is altered ${ }^{16}$, also complemented the inhibition of cell proliferation after $\mathrm{NEK} 9 \mathrm{KD}$, suggesting the possibility that kinase activity is not required for the growth-promoting function (Fig. 2F and G). Fourth, we evaluated the effect of a NEK9 siRNA/ drug-delivery system (DDS) carrier complex on cell proliferation in p53-mutant xenograft tumors consisting of SW480 cells, derived from a p53-mutant human colon cancer, in mice. As indicated in Fig. 3A, the siRNA/DDS carrier complex was administered into transplanted tumors five times at 2-day intervals, and tumor size was measured at every 2 days. In these animals, NEK9 KD markedly inhibited in vivo growth of p53 mutant cancer cells, but not of p53 WT cells (Fig. 3B, C, and D), providing further evidence that NEK9 contributes to cell proliferation in p53-mutant cancer cells. Taken together, these results clearly indicate that p53 deficiency makes cells dependent on NEK9 for proliferation, both in vitro and in vivo.

Inhibition of G1-S progression and induction of senescence-like features by NEK9 KD. Because NEK9 regulates proper positioning of bipolar spindles ${ }^{17-20}$, we hypothesized that NEK9 KD prevents G2/ $\mathrm{M}$ progression. In flow-cytometry analysis, however, NEK9 KD had 


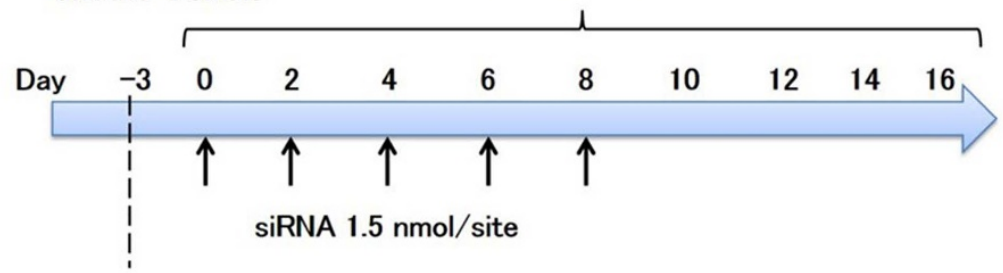

\section{HCT116 or SW480}

$5.0 \times 10^{5}$ cells $/$ site
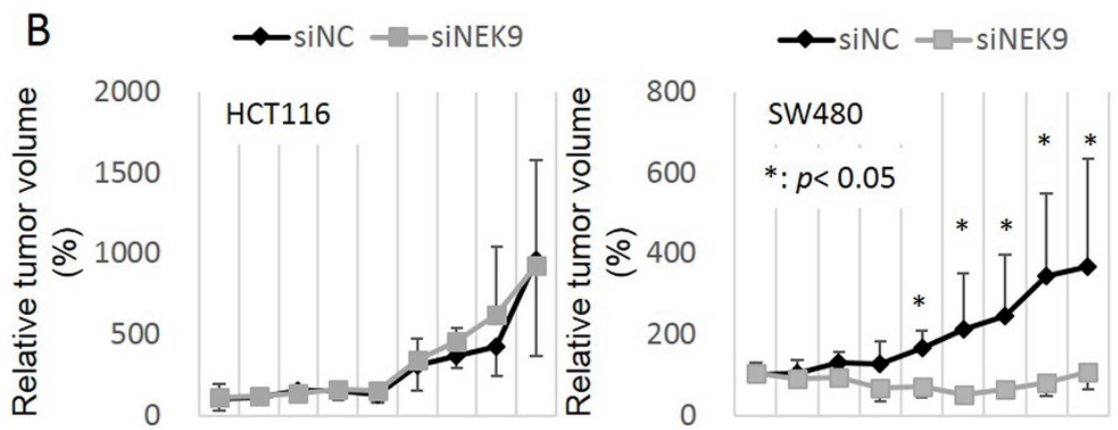

0
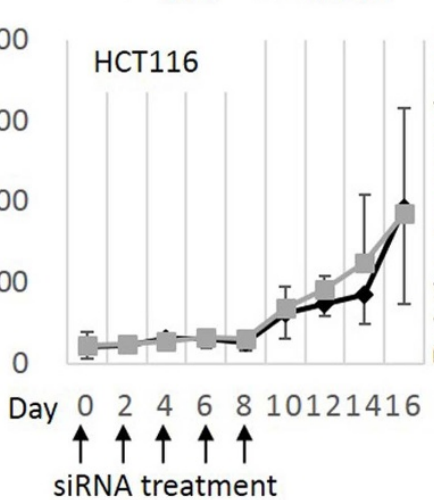

C
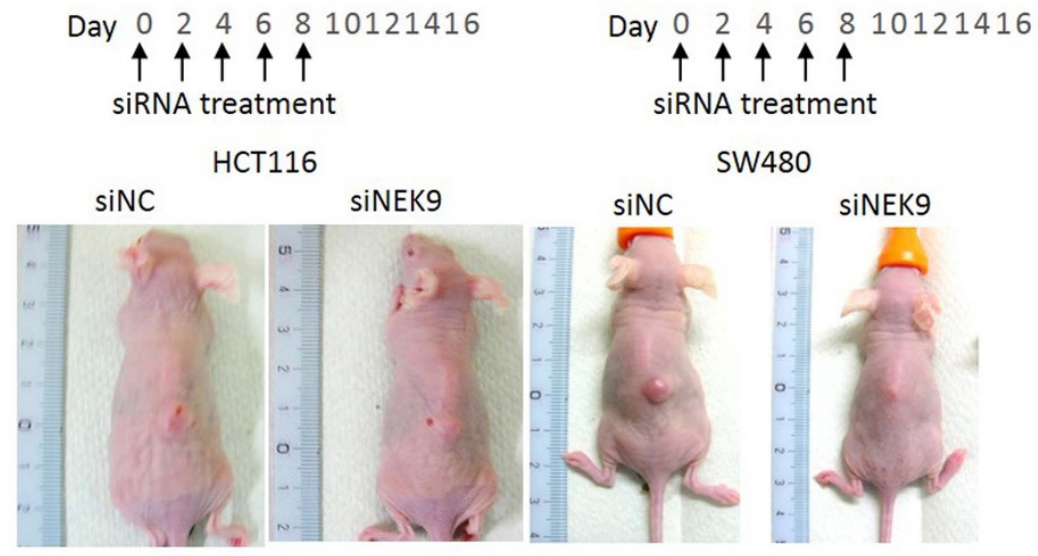

siRNA treatment

SW480

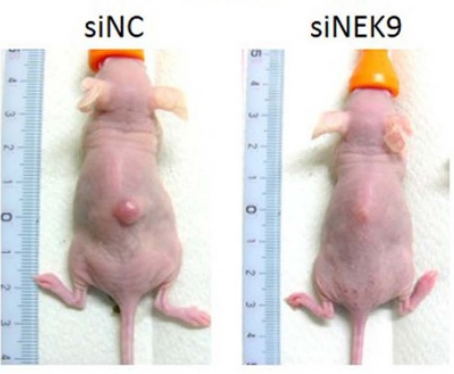

D

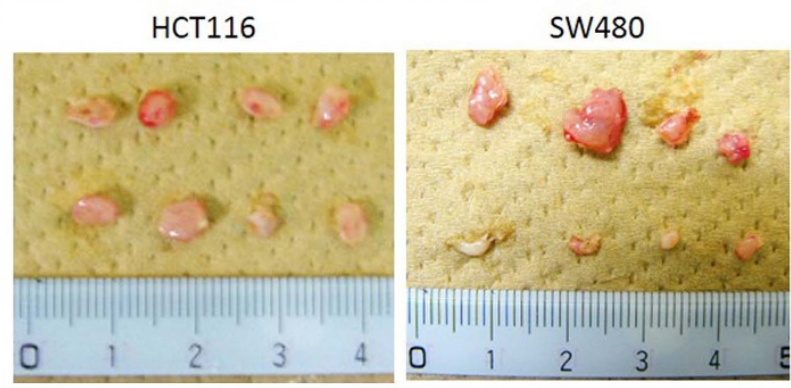

siNC

SiNEK9

Figure 3 Growth inhibition of p53-mutant xenograft by NEK9 siRNA. (A) Schedule of administration of NEK9 siRNA into SW480 or HCT 116 xenografts. Arrows show the days on which injections of siRNA/in vivo-jetPEI complex were administered. (B) SW480 and HCT 116 cells were inoculated into the backs of nude mice $(\mathrm{n}=4)$, and siRNAs (NEK9 or negative control $[\mathrm{NC}]$ ) in complex with drug-delivery carrier were injected on the specified days (arrows at the bottom of the graph). Tumor volume is expressed as a percentage (\%) relative to the volume at the time of the first siRNA injection (day 0). Error bars indicate SD $(n=4)$. A left graph indicates tumor growth of p53 WT HCT 116 cells. A right graph is p53 mutant SW480.

(C) Representative images of mice with xenograft tumors are shown on the left (HCT 116) and right (SW480). (D) Comparison of xenograft tumors injected with NC (upper panel) and NEK9 siRNA (lower panel).

no significant effect on the fraction of cells in G2/M phase; rather, in the two p53-mutant cell lines SW480 and PANC1, NEK9 KD significantly increased the G1-phase population while significantly decreasing the S-phase population (Fig. 4A). We obtained similar results in H1299 transfectants expressing p53 mutants, as well as in p53 KD HCT116 cells (Supplementary Figure 4). These data indicated that NEK9 is necessary for the G1-S transition in p53deficient cancer cells.

The growth arrest resulting from NEK9 KD was associated with flattened and enlarged cellular morphologies typical of cellular senescence. Consistent with this, the cells stained positively for senescence-associated $\beta$-galactosidase (SA- $\beta$-gal) (Fig. 4B). Flow- 


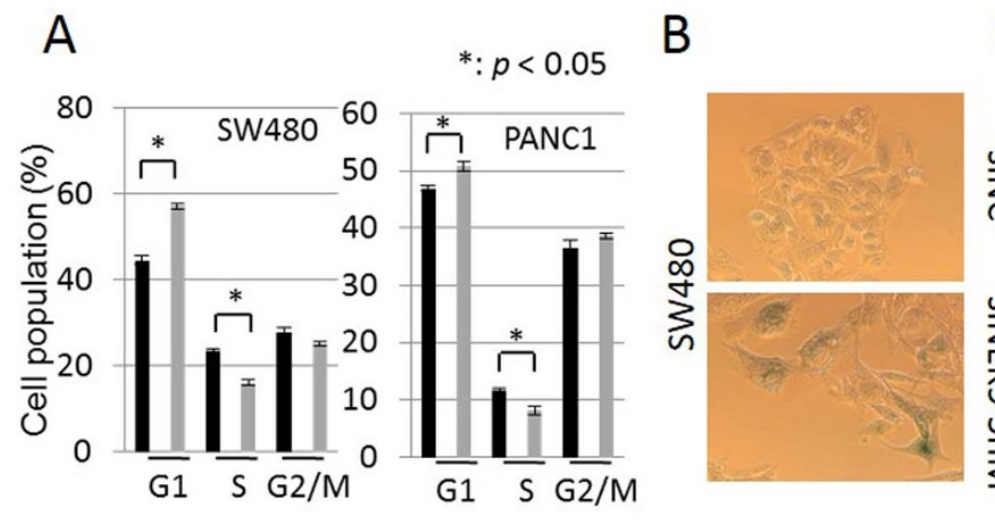

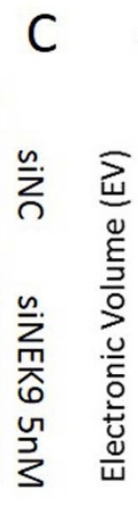

D

$\mathrm{E}$

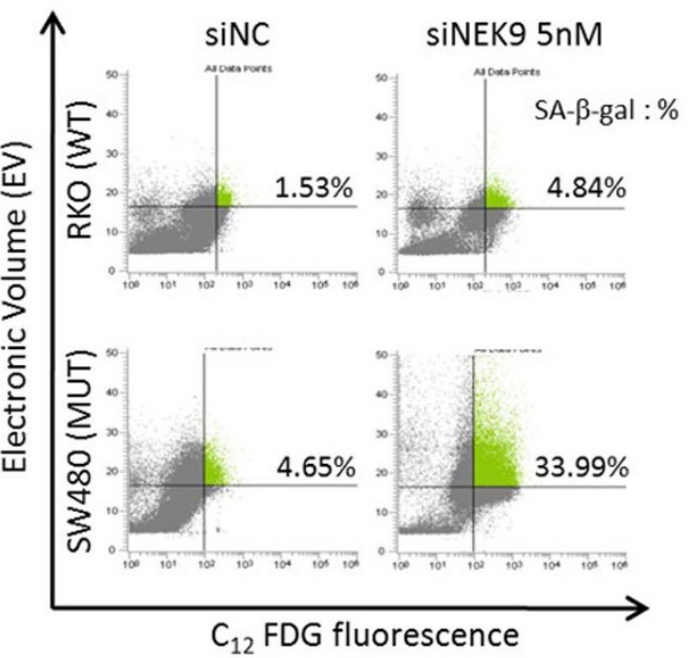

\section{$\frac{\cup}{\text { 紊 }}$}

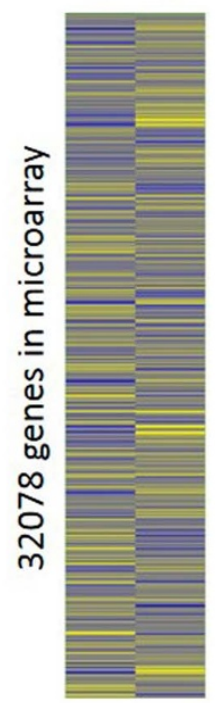

Up-regulating genes Both MUT and KO Compared with WT

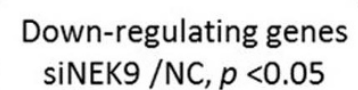

Down-regulating genes

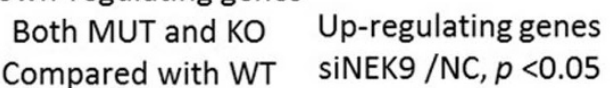

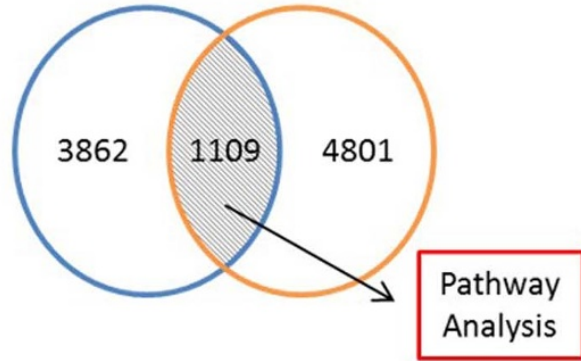

$\mathrm{F}$
RKO

SW480
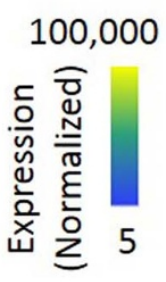

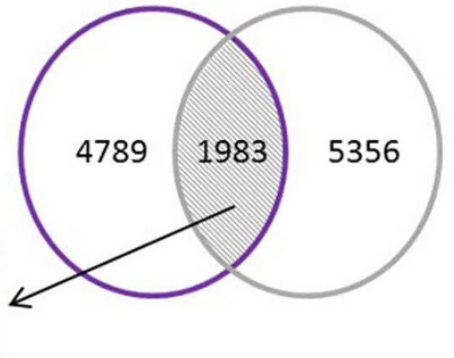

PANC1

\begin{tabular}{|c|c|c|c|}
\hline$+\cdots$ & $\because \cdots$ & O- & NEK9 \\
\hline & $1+6$ & $\approx \infty$ & p21 \\
\hline & $\mid=--\infty$ & $-\cdots$ & MAPK14 \\
\hline$-\pi$ & E & {$[5$} & Total \\
\hline
\end{tabular}

Figure $4 \mid$ NEK9 KD induces G1 arrest with senescence-like features and broad changes in gene expression profile. (A) Seventy-two hours after transfection with $2 \mathrm{nM}$ siRNA, cells were subjected to flow-cytometry analysis. Error bars indicate SD of three independent experiments. (B) SW480 cells were transfected with $5 \mathrm{nM}$ siRNAs and incubated for 7 days. (C) SA- $\beta$-gal-positive cells were quantified by flow cytometry using $\mathrm{C}_{12} \mathrm{FDG}$ as a fluorogenic substrate. The upper panels show data from RKO (p53 WT), and the lower panels show data from SW480 (p53 MUT). Electronic volume (EV) indicates cell size. (D) Heat map of changes in mRNA levels $72 \mathrm{~h}$ after transfection of SW480 cells with $5 \mathrm{nM}$ siRNAs. (E) Venn diagrams indicate the number of genes up- and down-regulated in $533 \mathrm{MUT} / \mathrm{KO}$ and NEK9 KD cells. The blue circle represents genes up-regulated in p53 mutant (MUT) and knockout (KO) cell lines relative to WT cells, and the orange circle represents genes down-regulated genes in NEK9 KD cells. The purple circle represents genes down-regulated in p53 MUT and KO cells relative to WT cells, and the silver circle represents genes up-regulated in NEK9 KD cells. (F) Cells were transfected with the specified concentrations of siRNAs, incubated for $72 \mathrm{~h}$, and then subjected to immunoblot analysis with the indicated antibodies. The original full scan images are available in Supplementary Figure 7.

cytometry analysis ${ }^{21}$ confirmed that SA- $\beta$-gal staining increased upon NEK9 depletion to a much greater extent in p53-mutant cells than in p53 WT cells (Fig. 4C). These results strongly suggest that depletion of NEK9 causes senescence-like phenotypic changes in p53-deficient cancer cells, because such cells require NEK9 function to progress from $\mathrm{G} 1$ to $\mathrm{S}$ phase.

Induction of broad changes in the gene expression profile upon NEK9 KD. To further elucidate the molecular connection between NEK9 depletion and cell-cycle arrest/senescence, we analyzed changes in mRNA expression profiles following NEK9 KD in p53MUT cells. NEK9 KD caused broad changes in gene expression in p53-deficient cells (Fig. 4D): about 25\% of genes up-regulated by NEK9 KD were expressed at significantly lower levels in p53 MUT and $\mathrm{KO}$ than in WT, suggesting that these genes were downregulated due to loss of functional p53 (Fig. 4E). On the other hand, about $20 \%$ of genes down-regulated by NEK9 KD were expressed at significantly higher levels in MUT and KO than in WT, suggesting that their expression was increased by loss of functional p53 (Fig. 4E). Thus, NEK9 appears to be involved in regulation of a wide range of signaling pathways required for proliferation in p53-deficient cells.

Next, we performed a gene network analysis, using sets of genes affected in opposite directions by NEK9 KD and p53 deficiency 
(Fig. 4E). This analysis revealed that NEK9 repression modulates multiple pathways, including receptor signaling cascades, mRNA processing, and cell-cycle control (Supplementary Tables 2 and 3). Upon NEK9 KD in p53-deficient cells, but not p53 WT cells, two crucial regulators of the cell cycle were differentially regulated: $\mathrm{p} 21$ was up-regulated, and MAPK14 (also known as p38 $\alpha$ ) was downregulated (Fig. 4F). These two factors control the cell cycle regardless of TP53 status $^{22-26}$. However, in cancer cells with p53 mutations, NEK9 KD modulated the expression of both factors, leading to cell-cycle arrest with characteristics of senescence. These findings reveal a novel role of NEK9: regulation of the cell cycle, via control of multiple genes, in cancer cells carrying p53 mutations.

NEK9 expression in human lung cancer specimens expressing mutant p53. To elucidate whether NEK9 dependency occurs in human tumors, we next analyzed the expression of NEK9 and p53 proteins in cancerous and non-cancerous cells in vivo by an immunohistochemical staining of tissue microarray. In this method, positive staining of p53 indicates the expression of mutant p53 protein ${ }^{27}$. NEK9 signal was absent or faint in non-cancerous lung epithelial cells (Supplementary Figure 5A and B). By contrast, NEK9 was frequently expressed in several types of lung cancer: 33/57 (57.9\%) of adenocarcinomas (ADC), 54/56 (96.4\%) of squamous cell carcinomas (SQC), 23/23 (100\%) of large cell neuroendocrine carcinomas (LCNEC), and 22/22 (100\%) of small cell carcinomas (SCLC) (Fig. 5A). NEK9 protein was detectable in cancer cells that stained either positively or negatively with an anti-p53 antibody, i.e., again cells harboring either mutant or WT TP53, respectively. In the ADC and SQC samples, NEK9 staining did not correlate with either histological differentiation or p53 status. Notably, however, half of ADCs (13/26), the vast majority of SQCs (28/30), and all LCNECs $(20 / 20)$ and SCLCs $(14 / 14)$ that were positive for p53 staining also stained positively for NEK9 (Fig. 5B), indicating that NEK9 dependency occurs in lung SQCs, LCNECs, and SCLCs with p53 mutations. In addition, Kaplan-Meier analysis demonstrated that NEK9/p53 double-positive cases had significantly poorer prognoses than other cases (Fig. 5C and Supplementary Figure 5C), suggesting that NEK9 confers a growth advantage on ADCs harboring p53 mutations, resulting in aggressive tumors with poor clinical outcomes. To extend and validate these results, we used microarrays to measure NEK9 mRNA levels in a different set of 76 lung ADC samples, in which TP53 mutations were defined by exome sequencing (Fig. 5D, Supplementary Table 4). In addition, we used The Cancer Genome Atlas (TCGA) ${ }^{28}$ data to analyze NEK9 expression levels in various cancers. As indicated in Fig. 5D and Supplementary Figure 5D, NEK9 mRNA was expressed at similar levels in ADCs and SQCs of the lung, colon, stomach, and serous ovarian cancers, regardless of TP53 status. Judging from these results, NEK9 dependency makes a considerable contribution to growth and/or progression of tumors harboring TP53 mutations.

\section{Discussion}

Through $m i R-22$ target screening, we identified NEK9 as a crucial regulator of cell-cycle progression in cancer cells lacking functional p53. The miRNA target screen is based on unique features of miRNAs, which target multiple mRNAs and induce phenotypes in a cell type-dependent manner. In a previous study, we showed that miR-22 induces apoptosis in p53 WT cancer cells, but causes cellcycle arrest in p53 MUT and KO cells that express CDKs and HDACs, critical regulators of the cell cycle that are potential targets of $m i R-22^{15}$. Therefore, we hypothesized that specific factor(s) repressed by $m i R-22$ are required for cell-cycle progression in cancer cells with p53 deficiencies. Consistent with this idea, our screen successfully identified NEK9 as a crucial factor involved in cell-cycle progression in p53-deficient cancer cells.
NEK9 regulates mitotic entry by phosphorylating two other NEK family proteins, NEK6 and $7^{17-20,29,30}$, and it is also activated sequentially by PLK1 and CDK1 to regulate formation of the mitotic spindle ${ }^{31-33}$. PLK1 was previously identified as a factor required for survival of isogenic p53 KO cell lines ${ }^{34}$. The results described here demonstrate that NEK9 inhibition does not affect the proportion of cells in G2/M phase, indicating that NEK9 is involved in molecular mechanism(s) distinct from those dependent on PLK1, at least in p53-deficient cancer cells. In fact, proliferation in p53 mutant cells was not specifically inhibited following knockdown of NEK6 or 7 (Supplementary Figure 6A and B).

NEK9 interacts with the FACT (facilitates chromosome transcription) complex in HeLa cells ${ }^{35}$, in which functional p53 is disrupted by a viral protein. Knockdown of FACT components inhibits progression from $\mathrm{G} 1$ to $S$ phase ${ }^{35}$. These findings are consistent with our own observation that NEK9 KD caused cell-cycle arrest at G1 phase and modulates the global gene expression profile in p53-deficient cells. Furthermore, phosphorylation of NEK9 at T210, which is required for activation of kinase activity ${ }^{16}$, is important for the interaction between NEK9 and FACT ${ }^{35}$. Our findings, however, demonstrated that expression of NEK9 T210A restored proliferation in p53 mutant cells, suggesting that the role of NEK9 in regulating G1-S progression is kinase-independent (Fig. 2G). Consistent with this, a previous report showed that phosphorylation at T210 and NEK9 kinase activity are only detectable during G2/M phase, and are required for G2 phase progression ${ }^{16}$. However, the possibility that an uncharacterized phosphorylation site(s) and/or an interaction of protein partner(s) is required for the kinase activity of NEK9 to regulate the progression of the cell cycle from G1 to $S$ phase in the p53-inactivated cells can not be excluded. Further studies are needed to clarify this important issue.

A recent study demonstrated that p53 mutations exert tumorpromoting functions that affect cell-cycle progression and metastatic activity in cancer cells ${ }^{36}$. Therefore, we hypothesized that NEK9 promotes these oncogenic functions of mutant p53. However, we did not detect an interaction between NEK9 and mutant p53 proteins (data not shown). Furthermore, NEK9 KD inhibited the proliferation of p53 KD cells (Fig. 2B). These observations suggest that NEK9 is associated with proliferation and cell-cycle progression in a manner that does not involve a direct interaction with p53. Currently, the relationships between NEK9, mutant $\mathrm{p} 53$, and the downstream targets of NEK9 remain unclear due to the unique functions of NEK9, including its kinase independent activity. Therefore, a more detailed analysis of NEK9 function will be required to identify the network(s) controlling the proliferation of p53-inactivated human cancer cells.

Mutant p53 proteins have attracted a great deal of attention as potential targets for cancer therapy. Based on this idea, several approaches have been attempted, including allele-specific activation of WT $\mathrm{p} 53$ and restoration of WT function to mutant p53 using small molecules ${ }^{37,38}$. Inhibition of NEK9 scaffolding (or NEK9 complex formation) and kinase functions may provide a means for selectively targeting cancer cells that lack functional p53; thus, our findings might lead to the development of novel cancer therapies.

\section{Methods}

Cell lines. Cell lines used for this study were obtained from the American Type Culture Collection (ATCC, Manassas, VA, USA). HCT116 p53 cells were kindly provided by Dr. Bert Vogelstein (The Johns Hopkins University, Baltimore, MD, USA). Colon and pancreatic cancer cell lines were cultured in Dulbecco's Modified Eagle's Medium (DMEM) supplemented with $10 \%$ heat inactivated fetal bovine serum (FBS), and lung cancer cell lines were maintained in RPMI-1640 (Gibco) supplemented with $10 \%$ FBS.

Microarray analyses. Microarray analysis was carried out as described previously ${ }^{15}$. In brief, SW480 or HCT116 p53 $3^{--}$cells, seeded at $5.0 \times 10^{4}$ cells $/ \mathrm{ml}$, were transfected for $48 \mathrm{~h}$ with either $m i R-22$ or miR-negative control (Ambion) at a final concentration of $5 \mathrm{nM}$ using HiPerFect Reagent (Qiagen). Total RNA was prepared using RNeasy columns (Qiagen) and labeled with Cy3 using the Low Input Quick Amp labeling kit (Agilent). Microarray analysis was performed using Whole Human 
A $A D C$
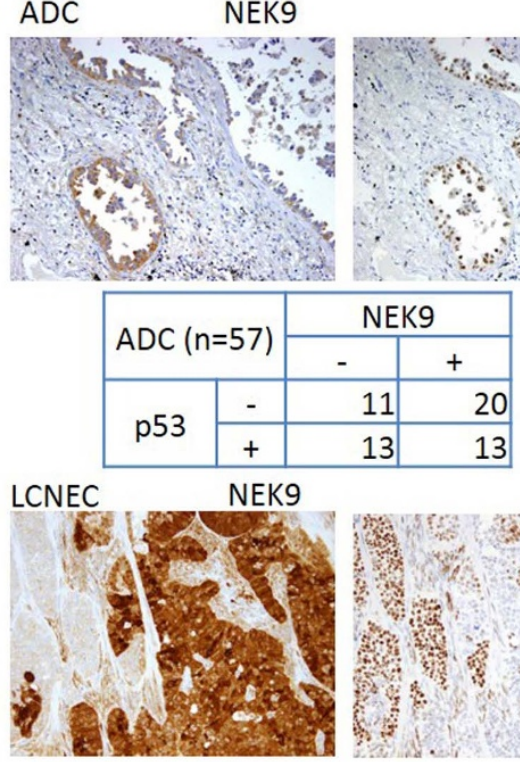

\begin{tabular}{|c|r|r|r|}
\hline \multicolumn{2}{|c|}{$\begin{array}{c}\text { LCNEC } \\
(n=23)\end{array}$} & \multicolumn{2}{|c|}{ NEK9 } \\
\cline { 2 - 4 } p53 & - & & \multicolumn{1}{c|}{+} \\
\cline { 2 - 4 } & + & 0 & 20 \\
\hline \multirow{2}{*}{ p }
\end{tabular}

p53 SQC

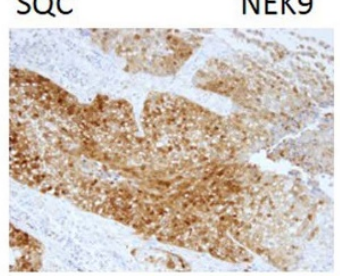

(x 20)

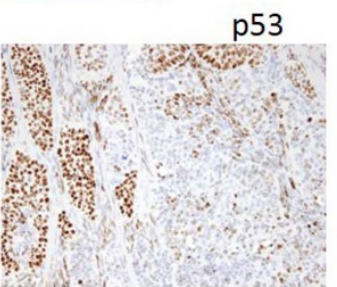

$(\times 20)$

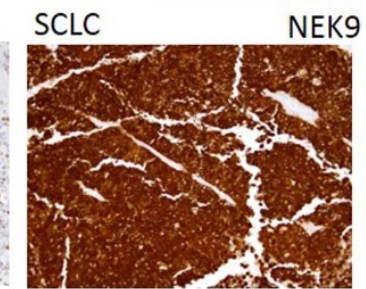

\begin{tabular}{|c|c|r|r|}
\hline \multirow{2}{*}{ SQC $(n=56)$} & \multicolumn{2}{|c|}{ NEK9 } \\
\cline { 3 - 4 } p53 & - & - & \multicolumn{1}{c|}{+} \\
\hline \multirow{2}{*}{ pyyy } & + & 2 & 26 \\
\hline
\end{tabular}

NEK9

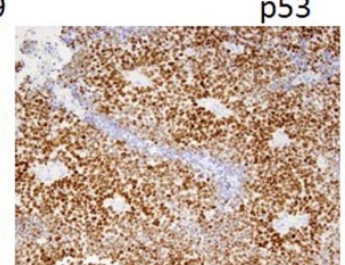

$(x 20)$

$(x 20)$

p53

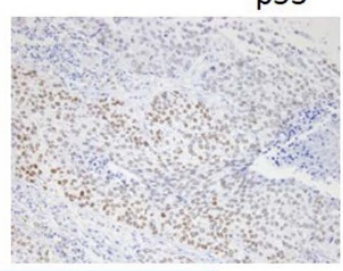

\begin{tabular}{|c|c|c|c|}
\hline \multirow{2}{*}{\multicolumn{2}{|c|}{$\operatorname{SCLC}(n=22)$}} & \multicolumn{2}{|c|}{ NEK9 } \\
\hline & & - & + \\
\hline \multirow{2}{*}{ p53 } & - & 0.0 & 8 \\
\hline & + & 0.0 & 14 \\
\hline
\end{tabular}

ADC

B

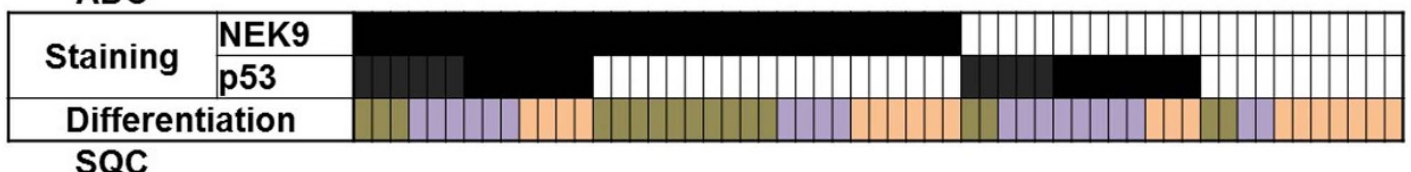

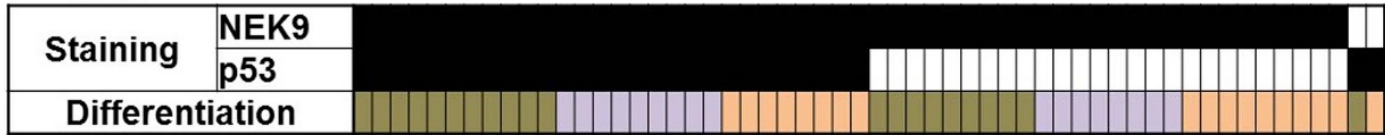

LCNEC

\begin{tabular}{|c|c|}
\hline Staining & \begin{tabular}{|l} 
NEK9 \\
p53
\end{tabular} \\
\hline SCLC & \\
\hline Staining & \begin{tabular}{|l} 
NEK9 \\
p53
\end{tabular} \\
\hline
\end{tabular}

C

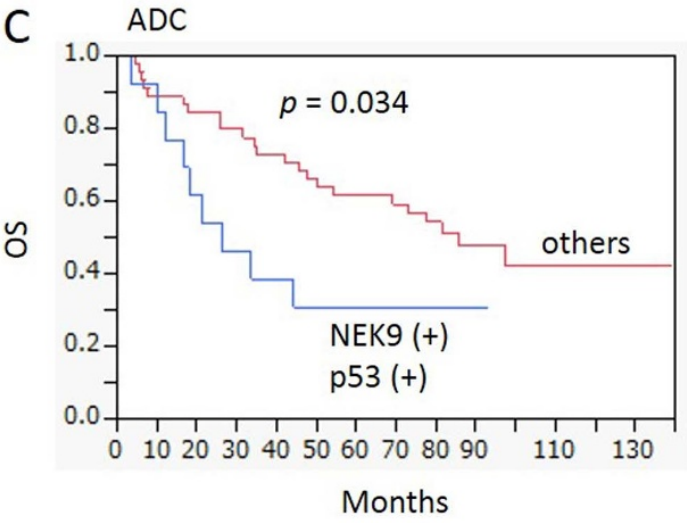

D
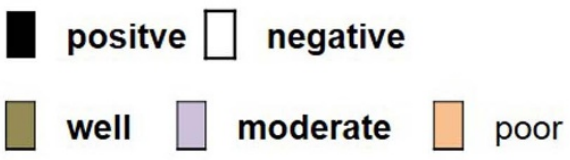

$A D C$

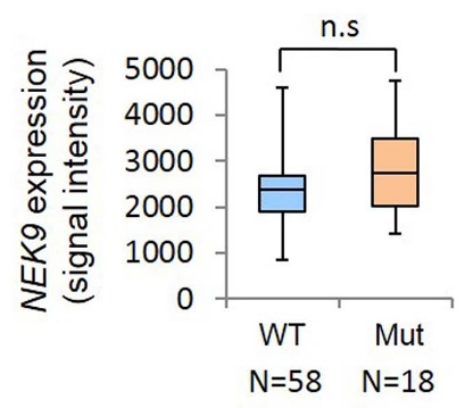

Figure 5 | Expression of NEK9 protein in lung cancer. (A) Representative images of NEK9 and p53 double-positive lung adenocarcinoma (ADC, upper left pair of images), squamous cell carcinoma (SQC, upper right pair), large cell neuroendocrine carcinoma (LCNEC, bottom left pair), and small cell lung carcinoma (SCLC, bottom right pair). The tables below each image pair show the number of samples of each type of cancer that contained positively stained cells. (B) NEK9- and p53-positive cases of ADC and SQC, with histological differentiation type indicated for each case. (C) KaplanMeier survival curves comparing NEK9 and p53 double-positive ADCs versus other types of ADCs. Vertical axis indicates overall survival (OS); horizontal axis indicates duration (months). Statistical analysis was carried out by Wilcoxon test. (D) Levels of NEK9 mRNA in human lung ADCs were determined by microarray analysis. TP53 mutation status was determined by whole-exome sequencing. Boxes represent the upper and lower quartiles and the median, and whiskers show the maximum and minimum values. 
Genome Oligo Microarrays $(4 \times 44 \mathrm{~K} v 2$, Agilent), and the data were analyzed using the GeneSpring GX11.5 software (Agilent).

For the generation of mRNA expression profiles in NEK9 KD cells, SW480 cells were transfected with NEK9 siRNAs, total RNAs were prepared, and microarray analysis was carried out as described above. Network analysis was performed using WikiPathways (http://wikipathways.org/).

Human tumor xenograft model. SW480 cells (derived from a p53-mutant human colon cancer) were inoculated at $5.0 \times 10^{5}$ cells per site on the backs of 6-week-old female nude mice (CLEA Japan, Inc.). in vivo-jetPEI (Polyplus Transfection)/siRNA complexes were prepared in a volume of $100 \mu \mathrm{l}(1.5 \mathrm{nmol} /$ site $)$ according to the manufacturer's instructions, and then injected intratumorally into each animal five times at 2-day intervals. Tumor size was monitored every 2 days by measuring the length $(\mathrm{L})$ and width $(\mathrm{W})$ with calipers, and volume $(\mathrm{V})$ was calculated according to the formula $\mathrm{V}=\left(\mathrm{L} \times \mathrm{W}^{2}\right) \times 0.5$. Tumor volume is expressed as percentage of the volume on the day of the first siRNA injection. The animal study protocol was approved by the Committee for Ethics in Animal Experimentation of the National Cancer Center (NCC) Research Institute, and the experiments were carried out according to the NCC guidelines.

Analysis of human lung cancer samples. Surgical specimens of primary human lung cancer were obtained from patients treated at the National Cancer Center (NCC) Hospital, with documented informed consent obtained in each case. Whole-exome sequence analysis was carried out using the Agilent SureSelect Human All-Exon kit, and sequencing was performed on the Illumina HiSeq 2000 platform. TP53 mutations were evaluated using sequencing data. Gene expression profiles were generated using Agilent Whole Human Genome DNA Microarrays. NEK9 expression levels in lung cancer were then determined.

Immunohistochemical analysis was conducted on tissue microarray sections derived from anonymized samples from patients with ADC, SQC, LCNEC, or SCLC of the lung who were treated in the NCC Hospital. Antibodies against NEK9 (rabbit monoclonal antibody, EP7361, from Epitomics) and p53 (mouse monoclonal antibody, DO-7, from Dako) were used for these analyses. Institutional review board approval for the use of clinical samples was obtained from the NCC.

Supplementary information. Supplementary information incudes extended Methods, eight figures and 5 tables.

1. Vousden, K. H. \& Prives, C. Blinded by the Light: The Growing Complexity of p53. Cell 137, 413-431 (2009).

2. Harris, S. L. \& Levine, A. J. The p53 pathway: positive and negative feedback loops. Oncogene 24, 2899-2908 (2005).

3. Watson, I. R., Takahashi, K., Futreal, P. A. \& Chin, L. Emerging patterns of somatic mutations in cancer. Nat Rev Genet 14, 703-718 (2013).

4. Muller, P. A. \& Vousden, K. H. p53 mutations in cancer. Nat Cell Biol 15, 2-8 (2013).

5. Vousden, K. H. \& Prives, C. P53 and prognosis: new insights and further complexity. Cell 120, 7-10 (2005).

6. Freed-Pastor, W. A. \& Prives, C. Mutant p53: one name, many proteins. Genes Dev 26, 1268-1286 (2012).

7. Bartel, D. P. MicroRNAs: genomics, biogenesis, mechanism, and function. Cell 116, 281-297 (2004).

8. Calin, G. A. \& Croce, C. M. MicroRNA signatures in human cancers. Nat Rev Cancer 6, 857-866 (2006).

9. van Kouwenhove, M. Kedde, M. \& Agami, R. MicroRNA regulation by RNAbinding proteins and its implications for cancer. Nat Rev Cancer 11, 644-656 (2011).

10. Hermeking, H. MicroRNAs in the p53 network: micromanagement of tumour suppression. Nat Rev Cancer 12, 613-626 (2012).

11. Lujambio, A. \& Lowe, S. W. The microcosmos of cancer. Nature 482, 347-355 (2012).

12. He, L. et al. A microRNA component of the p53 tumour suppressor network. Nature 447, 1130-1134 (2007).

13. Raver-Shapira, N. et al. Transcriptional activation of miR-34a contributes to p53mediated apoptosis. Mol Cell 26, 731-743 (2007).

14. Tazawa, H., Tsuchiya, N., Izumiya, M. \& Nakagama, H. Tumor-suppressive miR34a induces senescence-like growth arrest through modulation of the E2F pathway in human colon cancer cells. Proc Natl Acad Sci U S A 104, 15472-15477 (2007).

15. Tsuchiya, N. et al. Tumor suppressor miR-22 determines p53-dependent cellular fate through post-transcriptional regulation of p21. Cancer Res 71, 4628-4639 (2011).

16. Tan, B. C. \& Lee, S. C. Nek9, a novel FACT-associated protein, modulates interphase progression. J Biol Chem 279, 9321-9330 (2004).

17. Belham, C. et al. A mitotic cascade of NIMA family kinases. Nercc1/Nek9 activates the Nek6 and Nek7 kinases. J Biol Chem 278, 34897-34909 (2003).

18. Richards, M. W. et al. An autoinhibitory tyrosine motif in the cell-cycle-regulated Nek7 kinase is released through binding of Nek9. Mol Cell 36, 560-570 (2009).

19. Sdelci, S., Bertran, M. T. \& Roig, J. Nek9, Nek6, Nek7 and the separation of centrosomes. Cell cycle 10, 3816-3817 (2011).
20. Yang, S. W. et al. Nek9 regulates spindle organization and cell cycle progression during mouse oocyte meiosis and its location in early embryo mitosis. Cell cycle 11, 4366-4377 (2012)

21. Debacq-Chainiaux, F., Erusalimsky, J. D., Campisi, J. \& Toussaint, O. Protocols to detect senescence-associated beta-galactosidase (SA-betagal) activity, a biomarker of senescent cells in culture and in vivo. Nature Protoc 4, 1798-1806 (2009).

22. McDonald, E. R. 3rd., Wu, G. S., Waldman, T. \& El-Deiry, W. S. Repair Defect in p21 WAF1/CIP1 -/- human cancer cells. Cancer Res 56, 2250-2255 (1996).

23. Abbas, T. \& Dutta, A. p21 in cancer: intricate networks and multiple activities. Nat Rev Cancer 9, 400-414 (2009).

24. Comes, F. et al. A novel cell type-specific role of p38alpha in the control of autophagy and cell death in colorectal cancer cells. Cell Death and differentiation 14, 693-702 (2007).

25. Wagner, E. F. \& Nebreda, A. R. Signal integration by JNK and p38 MAPK pathways in cancer development. Nat Rev Cancer 9, 537-549 (2009).

26. Paillas, S. et al. MAPK14/p38alpha confers irinotecan resistance to TP53-defective cells by inducing survival autophagy. Autophagy 8, 1098-1112 (2012).

27. Iggo, R., Gatter, K., Bartek, J., Lane, D. \& Harris, A. L. Increased expression of mutant forms of p53 oncogene in primary lung cancer. Lancet 335, 675-679 (1990).

28. Kandoth, C. et al. Mutational landscape and significance across 12 major cancer types. Nature 502, 333-339 (2013).

29. Roig, J., Mikhailov, A., Belham, C. \& Avruch, J. Nercc1, a mammalian NIMAfamily kinase, binds the Ran GTPase and regulates mitotic progression. Genes Dev 16, 1640-1658 (2002).

30. O’Regan, L. \& Fry, A. M. The Nek6 and Nek7 protein kinases are required for robust mitotic spindle formation and cytokinesis. Mol Cell Biol 29, 3975-3990 (2009).

31. Rapley, J. et al. The NIMA-family kinase Nek6 phosphorylates the kinesin Eg5 at a novel site necessary for mitotic spindle formation. J Cell Sci 121, 3912-3921 (2008).

32. Bertran, M. T. et al. Nek9 is a Plk1-activated kinase that controls early centrosome separation through Nek6/7 and Eg5. EMBO J 30, 2634-2647 (2011).

33. Sdelci, S., Bertran, M. T. \& Roig, J. Nek9, Nek6, Nek7 and the separation of centrosomes. Cell cycle 10, 3816-3817 (2011).

34. Sur, S. et al. A panel of isogenic human cancer cells suggests a therapeutic approach for cancers with inactivated p53. Proc Natl Acad Sci U S A 106, 3964-3969 (2009).

35. Roig, J., Groen, A., Caldwell, J. \& Avruch, J. Active Nerccl protein kinase concentrates at centrosomes early in mitosis and is necessary for proper spindle assembly. Mol Biol Cell 16, 4827-4840 (2005).

36. Muller, P. A. \& Vousden, K. H. Mutant p53 in Cancer: New Functions and Therapeutic Opportunities. Cancer Cell 25, 304-317 (2014).

37. Brown, C. J., Lain, S., Verma, C. S., Fersht, A. R. \& Lane, D. P. Awakening guardian angels: drugging the p53 pathway. Nat Rev Cancer 9, 862-873 (2009).

38. Cheok, C. F., Verma, C. S., Baselga, J. \& Lane, D. P. Translating p53 into the clinic. Nat Rev Clin Oncol 8, 25-37 (2011).

\section{Acknowledgments}

This work was supported by the Advanced Research for Medical Products Mining Program of the National Institute of Biomedical Innovation (NIBIO, 08-02, 12-01), and by Grants-in-Aid from the Ministry of Health, Labor, and Welfare of Japan; the Ministry of Education, Culture, Sports, Technology of Japan; and the National Cancer Center (NCC) Research and Development Fund (to N. T.). Whole-exome sequencing and expression analysis of clinical samples were supported by $\operatorname{NIBIO}(10-41,10-43)$. We also thank the core facility of NCCRI for microarray analysis and animal experiments, which were supported by the NCC Research and Development Fund (23-A-7).

\section{Author contributions}

D.K. and N.T. designed all research, performed experiments, analyzed data, and wrote the paper. F.T. and T.O. carried out some experiments. M.W. and H.N. analyzed some data. H.S., K.M., J.Y. and T.K. performed expression and sequencing analyses of clinical samples. K.T. and S.W. performed clinical and pathological analyses.

\section{Additional information}

Supplementary information accompanies this paper at http://www.nature.com/ scientificreports

Competing financial interests: The authors declare no competing financial interests. How to cite this article: Kurioka, D. et al. NEK9-dependent proliferation of cancer cells lacking functional p53. Sci. Rep. 4, 6111; DOI:10.1038/srep06111 (2014).

This work is licensed under a Creative Commons Attribution-NonCommercialNoDerivs 4.0 International License. The images or other third party material in this article are included in the article's Creative Commons license, unless indicated otherwise in the credit line; if the material is not included under the Creative Commons license, users will need to obtain permission from the license holder in order to reproduce the material. To view a copy of this license, visit http:// creativecommons.org/licenses/by-nc-nd/4.0/ 